\title{
CKD increases the risk of atrial fibrillation
}

An analysis of the Atherosclerosis Risk in Communities (ARIC) cohort shows that chronic kidney disease (CKD) is associated with an increased risk of atrial fibrillation (AF). "Determining whether $\mathrm{CKD}$ is a risk factor for AF is relevant because first, it would provide additional evidence on the cardiovascular effects of CKD and second, it would suggest that prevention and treatment of CKD could also lead to prevention of AF," says corresponding author Alvaro Alonso.

Patients with CKD are known to be at increased risk of cardiovascular events. However, conflicting results from earlier prospective population-based studies on the association between kidney function and AF led the ARIC investigators to examine the association between kidney function and urinary albumin excretion and the incidence of AF. During the ARIC study, serum levels of creatinine and cystatin $\mathrm{C}$ were measured and estimated glomerular filtration rate (eGFR) was calculated using the CKD-EPI equation based on both these parameters. Urinary albumin and creatinine excretion measurements were also collected to determine the albumin:creatinine ratio (ACR).

From the cohort of 10,328 individuals for whom data were available, 788 incident cases of AF were identified during the 10-year follow-up period. After adjustment for potential confounders and a history of cardiovascular disease, the incidence of $\mathrm{AF}$ in individuals with an eGFR $_{\text {cys }} 15-29 \mathrm{ml} / \mathrm{min} / 1.73 \mathrm{~m}^{2}$ was three times higher than in individuals with an $\mathrm{eGFR}_{\text {cys }} \geq 90 \mathrm{ml} / \mathrm{min} / 1.73 \mathrm{~m}^{2}$. The risk of AF was two times higher in individuals with microalbuminuria $(30-299 \mathrm{mg} / \mathrm{g})$ than in those with an ACR of $<30 \mathrm{mg} / \mathrm{g}$, and macroalbuminuria ( $\geq 300 \mathrm{mg} / \mathrm{g}$ ) was associated with a hazard ratio of 3.2. Individuals with a combination of low $\mathrm{eGFR}_{\text {cys }}$ and macroalbuminuria were at the highest risk of AF: the hazard ratio in these individuals was 13.1 compared with those who had a normal ACR and optimal eGFR. eGFR cys $_{\text {had a more }}$ linear association with the incidence of AF than did eGFR ${ }_{\text {creat }}$, which had a J-shaped association. The associations were independent of age, sex, race, history of cardiovascular disease, use of antihypertensive medication or history of hypertension.

Although AF was mainly identified based on hospitalization discharge codes, which might have led to ascertainment bias, the study has important strengths. "Because of the large sample size and the availability of different measures of kidney function and damage, we were able to provide a more precise assessment of the

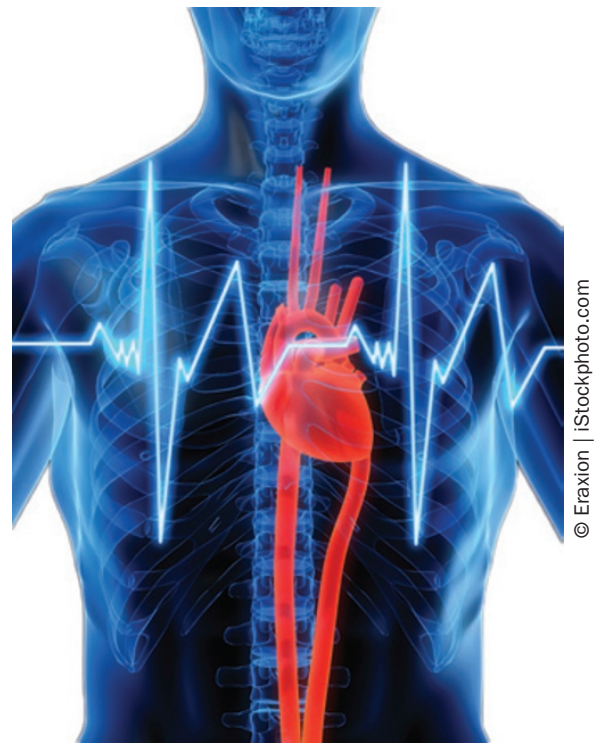

AF risk associated with CKD categories than previous studies," says Alonso. "Our results confirm observations from crosssectional studies in a prospective setting within a relatively large cohort. In terms of implications for patient care, our research suggests that it might be necessary to increase surveillance of AF in patients with CKD, particularly in those with other risk factors for AF."

Helene Myrvang

Original article Alonso, A. et al. Chronic kidney disease is associated with the incidence of atrial fibrillation: the Atherosclerosis Risk in Communities (ARIC) study. Circulation doi:10.1161/CIRCULATIONAHA.111.020982 